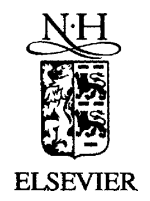

Information Sciences 149 (2003) 69-73

INFORMATION

SCIENCES

AN INTERNATIONAL JOURNAL

www.elsevier.com/locate/ins

\title{
Transparent silica glasses containing single walled carbon nanotubes
}

\author{
J. DiMaio, S. Rhyne, Z. Yang, K. Fu, R. Czerw, J. Xu, \\ S. Webster, Y.-P. Sun, D.L. Carroll, J. Ballato *
}

School of Materials Science and Engineering, Department of Physics and Astronomy, Department of Chemistry, Center for Optical Materials Science and Engineering Technologies, Clemson University, Clemson, SC 29634, USA

Received 27 September 2001; accepted 8 May 2002

\begin{abstract}
Organic/inorganic matrix nanocomposites have been created using an acid catalyzed, tetraethyl orthosilicate-based sol-gel technique with single-walled carbon nanotubes (SWNTs). By utilizing nanotubes functionalized with the dendron methyl 3,5-di(methyltrigycoloxy)benzylic alcohol $\left(\mathrm{I}_{\mathrm{PEG}}\right)$, ultrasonic blending in the sol phase prior to gelation yields excellent dispersion characteristics of the nanotube phase. The glasses were densified by heating to $600{ }^{\circ} \mathrm{C}$ yielding $80 \%$ of theoretical density with little change in the optical appearance or behavior. These materials exhibited intrinsic Rayleigh scattering, suggesting near ideal dispersion. Nonlinear optical transmission was observed for 1064 and $532 \mathrm{~nm}$ light suggesting that the matrix has a strong broad band coupling to the optical field. Such composites allow for a host of applications based on the novel confinement properties of carbon nanotubes in a robust inorganic host.
\end{abstract}

(c) 2002 Elsevier Science Inc. All rights reserved.

\section{Introduction}

Modification of optical properties in glasses through the introduction of nanosized materials holds much promise in a number of photonic applications

\footnotetext{
${ }^{*}$ Corresponding author. Present address: Department of Ceramic and Materials Engineering, Clemson University, Clemson, SC 29634-0907, USA.

E-mail address: john.ballato@ces.clemson.edu (J. Ballato).
} 
including nonlinear optics, optical switching, optical protection, spatial, and temporal filtering applications. Clearly, low dimensional metals and semiconductors offer unique optical properties when compared to bulk due to their localized band structure. Towards this end, single-walled carbon nanotubes (SWNTs) are receiving ever-increasing attention. Unfortunately, the realization of many desired optical effects relies on the character of the dispersion of the nanotubes, which has traditionally been quite poor. This is because aggregation within the matrix leads to significant Rayleigh scattering masking any unique optical property brought to the composite by the individual nanotubes.

In this work we describe preliminary efforts in using chemically functionalized SWNTs to permit their dispersion into an aqueous solution that then undergoes a hydrolysis and condensation reaction into a viscoelastic inorganic gel. Heat treatments then sinter this gel into a robust monolithic solid. Optical scattering and electron microscopy indicate that the nanotubes within the host glass with little signs of nanotube agglomeration. Further, nonlinear optical transmissivity is found at both 532 and $1064 \mathrm{~nm}$. This NLO response is observed for quite low loadings and is suggestive of the unique properties exhibited by the nanocomposites as they are only present in the glasses containing the carbon nanotubes.

\section{Experimental procedure}

SWNTs were produced by an arc discharge apparatus (Krätchmer generator) using $\mathrm{Ni}-\mathrm{Y}$ catalysts (yielding a diameter distribution of approximately $1.4-2.0 \mathrm{~nm}$ at lengths of approximately 1 micron). Purification via refluxing in aqueous $\mathrm{HNO}_{3}$ solution, as has reported in the literature was then used to remove the catalysts, leaving the nanotubes shorten to approximately $300-500$ $\mathrm{nm}$ [1]. Following acid treatment the nanotubes were repeatedly washed with water until neutral in $\mathrm{pH}$ and then dried. Purified samples were characterized by Raman spectroscopy, transmission electron microscopy (TEM), and scanning electron microscopy (SEM).

The full chemical route to functionalization is described in more detail elsewhere. ${ }^{1}$ However, in general, the dendron methyl 3,5-di(methyltriglycoloxy)benzylic alcohol (I $\mathbf{I}_{\mathbf{P E G}}$ ) was prepared first by mixing methyl 3,5-dihydroxybenzoate, triphenyl phosphine, and triethylene glycol monomethyl ether in anhydrous tetrahydrofurane, THF. Diethyl azodicarboxylate was added and the yellowish solution was stirred for $24 \mathrm{~h}$. After concentration and filtration, followed by silica gel column chromatography, the compound methyl 3,5-di(methyltriglycoloxy)benzoate was obtained and then chemically reduced using $\mathrm{LiAlH}_{4}$ to yield $\mathbf{I}_{\mathbf{P E G}}(95 \%$ yield $)$.

\footnotetext{
${ }^{1}$ To be published.
} 
The nanotubes were then functionalized by first treating in concentrated $\mathrm{HCl}$ solution to recover fully the carboxylic acid groups on the nanotube surface. After refluxing in thionyl chloride for $24 \mathrm{~h}$, the thionyl chloride-treated SWNT sample was added to a solution of $\mathbf{I}_{\mathbf{P E G}}$ in anhydrous THF mixed with $\mathrm{NaH}$. The reaction mixture was vigorously stirred under nitrogen for $24 \mathrm{~h}$, followed by repeated extraction with chloroform. The dark colored chloroform solution was briefly washed with water, followed by the solvent removal on a rotary evaporator to yield $\mathbf{I}_{\mathbf{P E G}}-\mathrm{SWNT}$ as a black solid. It was redissolved in deionized water for further purification via dialysis for 5 days.

The SWNTs were dispersed into an aqueous solution that then undergoes a hydrolysis and condensation reaction yielding a viscoelastic inorganic gel. Heat treatments then sinter this gel into a robust monolithic solid.

The composites were irradiated by a Nd:YAG laser $(532 / 1064 \mathrm{~nm})$ with a pulse width of 7-9 ns. Due to the composite thickness $(3-5 \mathrm{~mm})$ a focusing geometry of $\mathrm{f} / 65$ was used to maintain a constant irradiance through the samples. The radiant energy density was varied with a $\lambda / 2$ waveplate-polarizer setup and the input energy was monitored with a reference detector arm identical to the sample arm. With this setup, energy densities are easily varied over several orders of magnitude.

In Fig. 1, a photograph of a $5 \mathrm{~mm} \times 5 \mathrm{~mm} \times 1 \mathrm{~cm}$ monolith is shown that possesses a nanotube loading of $0.25 \%$ by weight. Samples were prepared over

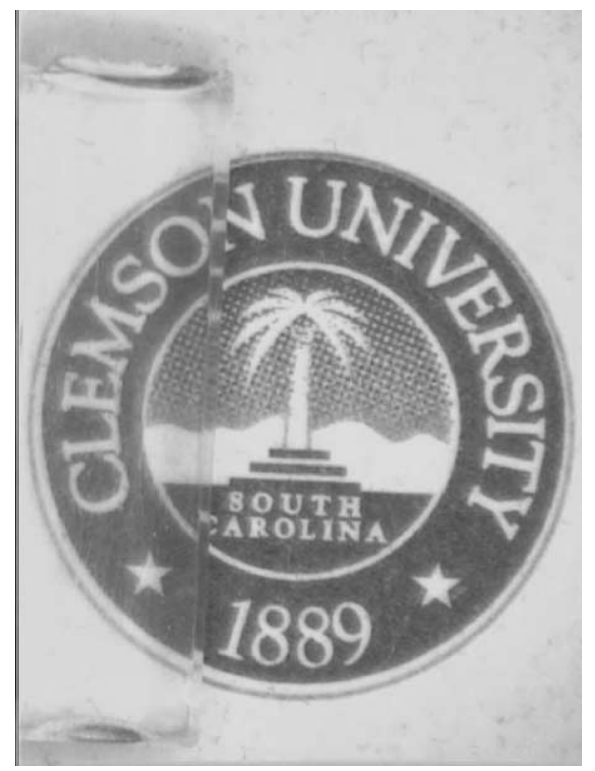

Fig. 1. Optical micrographs of the $0.25 \mathrm{wt} . \%$ nanotube-filled glasses are striking in their clarity. 
the wide range from $0.1 \%$ to $1.0 \%$ SWNTs-to- $\mathrm{SiO}_{2}$ by weight. Notice the clarity of the glasses. While it is reasonable to assume that a supersaturation phase exists in the initial solutions, clearly it is not reached at these loading levels. The glasses exhibit a distinct reddish color and we note that some color variations can be seen near the edges of the monoliths though these are exceedingly small. Further, the clarity of these materials is quite stable in air suggesting that the nanotube additions have little influence on thermomechanical behavior of the dense matrix

Fig. 2 shows the onset of nonlinear transmission from the nanotube/glass composite. The onset of nonlinearity is quite low for the materials are compared with other optical limiters [2]. Here is found an onset for limiting around $0.25 \mathrm{~J} / \mathrm{cm}^{2}$ for the $0.5 \%$ loaded glasses. Further. At these intensities, no optical damage was found either by observation under a microscope or from reproduction of the experiments. The origin of the limiting phenomena is still under investigation but is known to be quite different from that of carbon black and bucky-balls [3]. Efforts continue into elucidating the exact limiting behavior of the nanotubes in the glass as well as the influence on performance of the tube length and composition (by means of impurity doping), for example. It is clear, however, that the incorporation of carbon nanotubes into a glass generate nonlinear optical phenomena that are not present in the undoped cases.

In summary, we have demonstrated the exciting new possibility of nanotube-glass nanocomposites for nonlinear optical materials. Excellent dispersion of the nanotubes has been achieved using unique functionalization chemistry

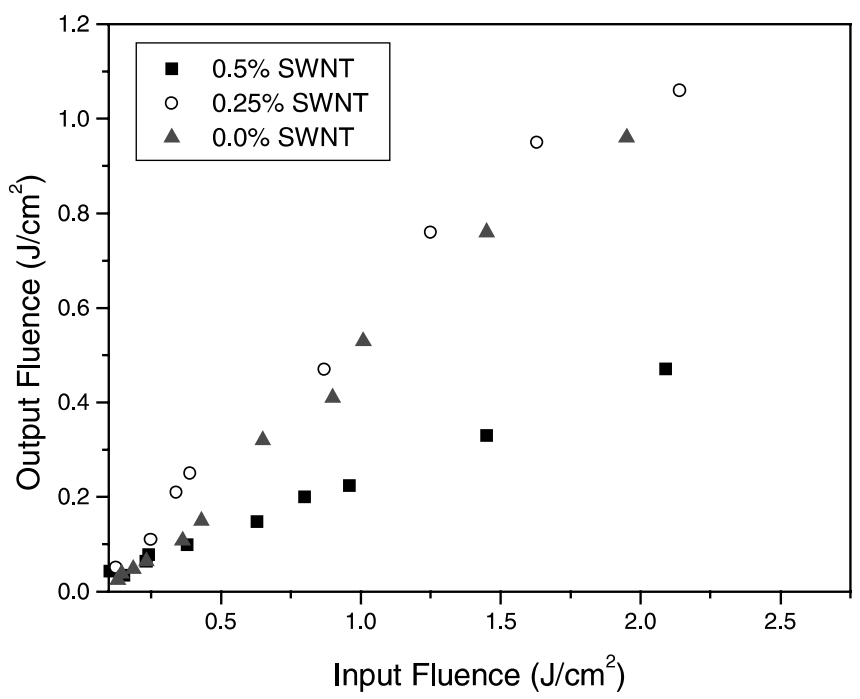

Fig. 2. Optical limiting at $1064 \mathrm{~nm}$ in the nanotube-glass as a function of nanotube loading level. 
with the carbon nanotubes. This opens many avenues of nonlinear optical applications of nanomaterials contained within a robust host matrix.

\section{Acknowledgements}

Financial support from AFOSR, NSF (CHE-9727506 and, in part, EPS9977797), NASA, ARO/DARPA (DAAD19-00-1-0002) through the MINSA program, 3M, and the South Carolina Space Grant Consortium is gratefully acknowledged.

\section{References}

[1] J. Liu, A.G. Rinzler, H. Dai, J.H. Hafner, R.K. Bradley, P.J. Boul, A. Lu, T. Lverson, K. Shelimov, C.B. Huffman, F. Rodriguez-Macias, Y.-S. Shon, T.R. Lee, D.T. Colbert, R.E. Smalley, Science 280 (1998) 1253-1256;

H. Hiura, T.W. Ebbesen, K. Tanigaki, Adv. Mater. 7 (1995) 275-276;

S.C. Tsang, Y.K. Chen, P.J.F. Harris, M.L.H. Green, Nature 372 (1994) 159-162;

A.G. Rinzler, J. Liu, H. Dai, P. Nikolaev, C.B. Huffman, F.J. Rodríguez-Macías, P.J. Boul, A.H. Lu, D. Heymann, D.T. Colbert, R.S. Lee, J.E. Fischer, A.M. Rao, P.C. Eklund, R.E. Smalley, Appl. Phys. A 67 (1998) 29-37.

[2] J.E. Riggs, D.B. Walker, D.L. Carroll, Y.P. Sun, J. Phys. Chem. B 104 (2000) 7071-7076.

[3] K. Mansour, M. Soileau, E. van Stryland, J. Opt. Soc. Am. B 9 (1992) 1100-1109. 